\title{
Tip motion in amplitude modulation (tapping-mode) atomic-force microscopy: Comparison between continuous and point-mass models
}

\author{
Tomás R. Rodríguez and Ricardo García ${ }^{a}$ \\ Instituto de Microelectrónica de Madrid, CSIC, Isaac Newton 8, 28760 Tres Cantos, Madrid, Spain
}

(Received 9 October 2001; accepted for publication 2 January 2002)

\begin{abstract}
We discuss the influence of high-order frequency components in the operation of an amplitude modulation atomic-force microscope (AFM). A comparative study of point-mass and continuous models is performed to describe the tip motion. The tip-surface interaction force excites high-order frequency components whenever a higher harmonic of the excitation force is close to an eigenmode of the cantilever beam. The strength of those components depends on the set point amplitude and the fundamental resonance frequency of the cantilever. However, for standard operating conditions with quality factors in the $10^{2}-10^{3}$ range, higher-order components are about three orders of magnitude smaller than the component at the excitation frequency. We conclude that point-mass models are suitable to describe the operation of a tapping-mode AFM in air environments. (C) 2002 American Institute of Physics. [DOI: 10.1063/1.1456543]
\end{abstract}

Amplitude modulation atomic-force microscopy (amAFM), also known as a tapping-mode AFM, is the most used scanning probe method for the characterization and modification of a variety of materials such as DNA, antibodies, polymers, and silicon surfaces. Several theoretical models have been proposed to explain and understand the tip motion. ${ }^{1-5}$ Those models considered the cantilever-tip ensemble as a point-mass spring with a single resonant frequency. The above models were successfully applied to explain some of the observed experimental behaviors such as the discontinuous transitions in amplitude curves, ${ }^{1,4,6}$ enhanced resolution with single proteins, ${ }^{7}$ liquid layers, ${ }^{8}$ or phase contrast in heterogeneous samples. ${ }^{9,10}$ Recently, several experimental contributions have emphasized the role of higher cantilever modes to obtain some material contrast. $^{11-13}$ Those experiments have suggested that more than one resonance should be considered to describe the tip motion in amplitude modulation AFM. Simultaneously, a few theoretical descriptions based on a continuous model have been developed to describe the higher oscillation modes of the cantilever. ${ }^{14}$

To shed more light into the relevance of higher oscillation modes in amplitude modulation AFM we have performed a comparative study between point-mass and continuous models. Some of the most common observables in am-AFM, such as cantilever-tip oscillation, its Fourier transform, and amplitude curves, have been calculated and compared.

The description of the tip motion with a point-mass model is performed with the theoretical framework provided by García and San Paulo. ${ }^{1}$ The continuous model considers the cantilever-tip ensemble simulated as a one-dimensional beam with a semispherical tip at the end. Long- and shortrange interaction forces are included in the model following the approximations given in Ref. 1.

The continuous model considers that the equation for the

${ }^{a)}$ Electronic mail: rgarcia@imm.cnm.csic.es dynamic deflection function $w(x, t)$ is described by ${ }^{14-16}$

$$
\begin{aligned}
& \frac{E I}{L^{4}} \frac{\partial^{4}}{\partial x^{4}}\left[w(x, t)+a_{1} \frac{\partial w(x, t)}{\partial t}\right]+b h \rho_{c} \frac{\partial^{2} w(x, t)}{\partial t^{2}} \\
& \quad=F_{\text {ext }}(x, t)+F_{\text {med }}+F_{t-s}(x, t) .
\end{aligned}
$$

$x$ is the coordinate along the beam, $E$ is the Young module, $I$ is the moment of inertia for a rectangular beam, $\rho_{c}$ is the mass density, and $a_{1}$ is the internal damping of the cantilever. $L, b$, and $h$ are, respectively, the length, width, and height of the cantilever. The boundary conditions consider that one end of the cantilever is clamped and the other is free. $F_{\text {ext }}, F_{\text {med }}$, and $F_{t-s}$ are the excitation, hydrodynamic damping with the medium, and interaction forces, respectively. The excitation force and the tip-surface force are applied at the free end of the beam. The dimensions of the beam $a_{1}, F_{\text {ext }}$, and $F_{\text {med }}$ are established in such a way that the amplitude versus frequency curve for the first mode is fitted to a Lorentzian for a single-harmonic oscillator with $R$, $f_{0}, k, A_{0}$, and $Q$ of $30 \mathrm{~nm}, 350 \mathrm{kHz}, 40 \mathrm{~N} / \mathrm{m}, 18.22 \mathrm{~nm}$, and 400 , respectively. Those values represent some of the most common cases for tapping-mode cantilevers and also the optimum conditions to minimize tip-sample damage while maximizing resolution in stiff materials.

To solve the above equation, the deflection is expressed in a base of the eigenmodes of the free cantilever. The above boundary conditions imply that the deflection can be separated into spatial and temporal components. Then, the initial equation is equivalent to a system of coupled anharmonic oscillators, one equation for each eigenmode. ${ }^{14}$ Then, we solve numerically the system of equations for the first three eigenmodes $(350.6,2197$, and $6153 \mathrm{kHz}$, respectively) with three coupled fourth-order Runge-Kutta algorithms.

Figure 1 shows the dependence of low- and highamplitude branches on the rest tip-surface separation for the continuous and point-mass models. At the scale of the image, both models provide identical results. The oscillation signal for low- and high-amplitude branches at a fixed tip-surface separation $z_{c}=14.5 \mathrm{~nm}$ is also plotted (inset). The oscillation 


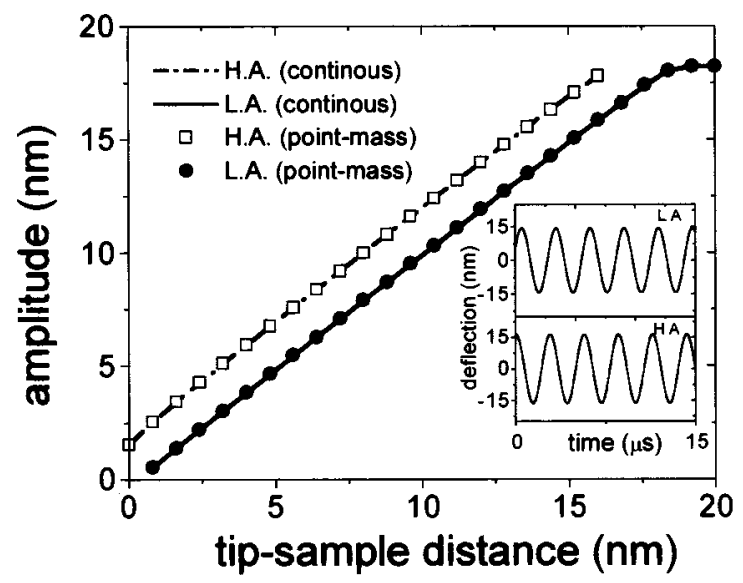

FIG. 1. Amplitude vs tip-surface distance curves for continuous (lines) and point-mass (symbols) models. Two different branches coexist. At this scale, both models give identical amplitude curves. The inset shows the deflection signal (high- and low-amplitude solutions) as a function of time for a fixed tip-surface separation $z_{c}=14.5 \mathrm{~nm}$ (continuous model).

signal is almost sinusoidal for both solutions. Again, at the scale of the image no differences are observed.

The almost sinusoidal behavior given by the point-mass model is better appreciated if the signal is discomposed in its frequency components (Fig. 2). A monotonous decay of the components of the amplitude with frequency is observed. The peak corresponding to the first harmonic is about 1700 times larger than the one corresponding to the second harmonic. No major differences are observed between $L$ and $H$ solutions. The presence of higher harmonic peaks is due to the strength of the tip-surface interaction force.

The continuous model reveals a qualitatively different result (Fig. 3). Although the oscillation signal shows an apparently sinusoidal behavior (inset, Fig. 1), the frequency domain shows a nonmonotonous decay. In addition to the peak at the excitation frequency, another local maximum appears at $\omega=6 \omega_{0}$. Other peaks appear at $\omega=17 \omega_{0}$ and $18 \omega_{0}$, respectively (not shown). Those peaks are a consequence of the existence of several oscillation modes of the cantilever. Whenever a higher harmonic of the excitation signal is close to the frequency of an eigenmode of the cantilever a local peak will be observed.

The strength of the interaction force enhances the effects of the eigenmodes of the cantilever. The peak at $6 \omega_{0}$ is twice higher in the $H$ branch than in the $L$ branch. The $H$ branch is

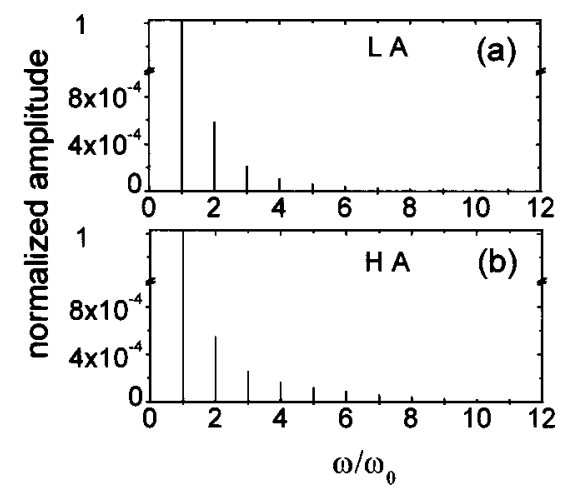

FIG. 2. Spectra of the oscillation signal calculated with the point-mass model. A monotonous decay of amplitude of higher harmonics is observed in both cases. (a) Low-oscillation state and (b) high-oscillation state. in both cases. (a) Low-oscillation state and (b) high-oscillation state. high-amplitude solution.
Downloaded 14 May 2002 to 150.244.37.101. Redistribution subject to AIP license or copyrgention

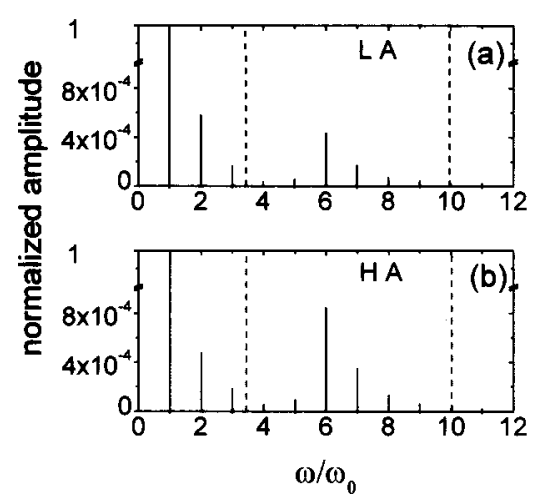

FIG. 3. Spectra of the oscillation signal calculated with the continuous model. The contributions of higher harmonics are modulated by the eigenmodes of the cantilever. (a) Low-oscillation state and (b) high-oscillation state. The vertical dashed lines show the rectangular bandpass filter applied to generate Fig. 4.

the result of attractive and repulsive forces while in the $L$ branch only attractive forces are present. Nevertheless, in both cases the second maximum is $10^{3}$ times smaller than the one corresponding to the fundamental mode.

It is important to observe that the most relevant experimental quantity, the oscillation amplitude, is effectively included in the amplitude of the fundamental peak. This result confirms the validity of point-mass models to describe amplitude modulation AFM experiments with relatively highquality factors, $Q \sim 10^{2}-10^{3}$.

Hillenbrand, Stark, and Guckenberger ${ }^{11}$ have suggested that information about the sample properties could be extracted from analysis of the higher harmonic components of the oscillation. To examine in more detail this aspect, in Fig. 4 we have plotted the result of applying a rectangular bandpass filter centered around the sixth harmonics to the oscillations shown in Fig. 3. A beat pattern is observed for both solutions $(L$ and $H$ ). The differences in the beat pattern reflect the strength of the interaction forces. However, this result only gives partial support to the Hillenbrand, Stark, and Guckenberger claims. For one thing, in the $L$ branch there is not a tip-sample mechanical contact, so only information about force gradients could be extracted. On the other hand, for the above operating conditions, the values of the highfrequency components are of the order of a few pm, barely in the measurable level.

The relative importance of higher harmonics in the tip motion depends on the value of the fundamental resonance frequency and the set point amplitude. The oscillation signal

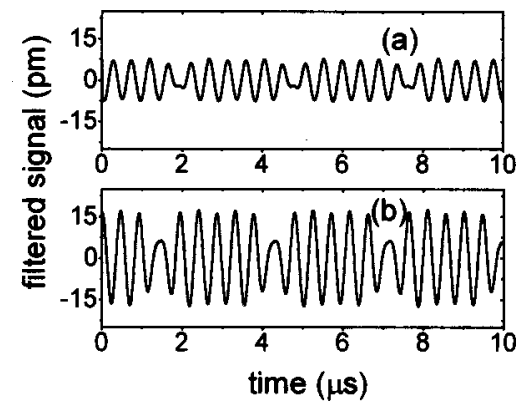

FIG. 4. Filtered oscillation signal obtained by the application of the rectangular bandpass filter shown in Fig. 3. (a) low-amplitude solution and (b) 
of a point-mass oscillator in the presence of a parabolic force $\left(F / m=-\epsilon z^{2}\right)$ can be approximated by

$$
z(t)=A \cos \left(\omega_{0} t\right)-\frac{\epsilon A^{2}}{\omega_{0}^{2}}\left(\frac{1}{2}-\frac{1}{6} \cos \left(2 \omega_{0} t\right)\right)+O\left(\epsilon^{2}\right),
$$

where $\epsilon$ is a measure of the interaction. Equation (2) shows that increasing the fundamental frequency decreases the contribution of higher harmonics, in this case, the second harmonic. This explains why for a $350 \mathrm{kHz}$ cantilever higher harmonic contributions are in the pm range while a $40 \mathrm{kHz}$ cantilever will give components in the $0.1 \mathrm{~nm}$ range for a set point amplitude of $20 \mathrm{~nm}$.

In short, the above results confirm and generalize for attractive and repulsive forces previous theoretical calculations (Ref. 14), which show that tip-surface interactions excite higher harmonics in the tip motion. Whenever a higher harmonic is close to an eigenmode of the cantilever an increase of the corresponding frequency component should be expected. However, in air environments $(Q \sim 100-1000)$ and $f_{0} \geqslant 10^{5} \mathrm{~Hz}$ higher frequency components are about three orders of magnitude smaller than the component of the fundamental frequency. As a consequence, simulations based on point-mass models are suitable to describe the cantilever tip motion in air. Higher harmonics contributions are expected to play a dominant role in low- $Q$ environments, i.e., in liquids where the cantilever spectra present broader and closer resonances.
The authors acknowledge fruitful discussions with A. San Paulo. This work was financially supported by the Dirección General de Investigación Científica y Técnica of Spain (PB98-0471).

${ }^{1}$ R. García and A. San Paulo, Phys. Rev. B 60, 4961 (1999).

${ }^{2}$ L. Nony, R. Boisgard, and J. Aimé, J. Chem. Phys. 111, 1615 (1999).

${ }^{3}$ M. Marth, D. Maier, J. Honerkamp, R. Brandsh, and G. Bar, J. Appl. Phys. 85, 7030 (1999).

${ }^{4}$ B. Gotsmann, C. Seidel, B. Anczykowski, and H. Fuchs, Phys. Rev. B 60, 11051 (1999).

${ }^{5}$ W. van der Water and J. Molenaar, Nanotechnology 11, 192 (2000).

${ }^{6}$ R. García and A. San Paulo, Phys. Rev. B 61, R13381 (2000).

${ }^{7}$ A. San Paulo and R. García, Biophys. J. 78, 1599 (2000).

${ }^{8}$ M. Luna, J. Colchero, and A. M. Baró, J. Phys. Chem. B 103, 9576 (1999).

${ }^{9}$ J. P. Cleveland, B. Anczykowski, A. E. Schmid, and V. Elings, Appl. Phys. Lett. 72, 2613 (1998).

${ }^{10}$ J. Tamayo and R. García, Appl. Phys. Lett. 71, 2394 (1998).

${ }^{11}$ R. Hillenbrand, M. Stark, and R. Guckenberger, Appl. Phys. Lett. 76, 3478 (2000).

${ }^{12}$ M. Stark, R. W. Stark, W. M. Heckl, and R. Guckenberger, Appl. Phys. Lett. 77, 3293 (2000).

${ }^{13}$ R. Stark, T. Drobek, and W. M. Heckl, Appl. Phys. Lett. 74, 3296 (1999).

${ }^{14}$ R. W. Stark and W. M. Heckl, Surf. Sci. 457, 219 (2000).

${ }^{15}$ H.-J. Butt and M. Jaschke, Nanotechnology 6, 1 (1995).

${ }^{16}$ L. D. Landau and E. M. Lifshitz, Theory of Elasticity (Pergamon, Oxford, 1970). 